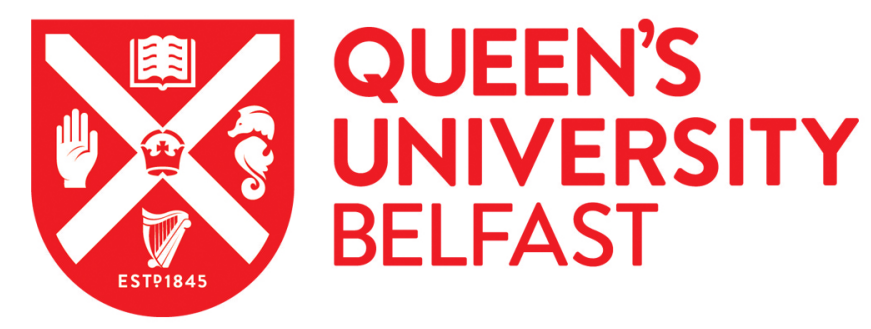

\title{
An international review and meta-analysis of prehabilitation compared to usual care for cancer patients
}

Treanor, C., Kyaw, T., \& Donnelly, M. (2017). An international review and meta-analysis of prehabilitation compared to usual care for cancer patients. Journal of Cancer Survivorship. https://doi.org/10.1007/s11764-0170645-9

Published in:

Journal of Cancer Survivorship

Document Version:

Peer reviewed version

Queen's University Belfast - Research Portal:

Link to publication record in Queen's University Belfast Research Portal

Publisher rights

Copyright 2017 Springer Verlag. This work is made available online in accordance with the publisher's policies. Please refer to any applicable terms of use of the publisher.

\section{General rights}

Copyright for the publications made accessible via the Queen's University Belfast Research Portal is retained by the author(s) and / or other copyright owners and it is a condition of accessing these publications that users recognise and abide by the legal requirements associated with these rights.

Take down policy

The Research Portal is Queen's institutional repository that provides access to Queen's research output. Every effort has been made to ensure that content in the Research Portal does not infringe any person's rights, or applicable UK laws. If you discover content in the Research Portal that you believe breaches copyright or violates any law, please contact openaccess@qub.ac.uk. 
Title: An international review and meta-analysis of prehabilitation compared to usual care for cancer patients

Authors: C. Treanor ${ }^{1}$, T. Kyaw ${ }^{1}$ and M. Donnelly ${ }^{1,2}$

Affiliation list: ${ }^{1}$ Centre for Public Health, School of Medicine, Dentistry and Biomedical Sciences, Queen's University Belfast, Belfast, Northern Ireland, United Kingdom; ${ }^{2}$ UKCRC Centre of Excellence for Public Health, School of Medicine, Dentistry and Biomedical Sciences, Queen's University Belfast, Belfast, Northern Ireland, United Kingdom

Corresponding author: Dr Charlene Treanor, Centre for Public Health, School of Medicine, Dentistry and Biomedical Sciences, Queen's University Belfast, Institute of Clinical Sciences-B Building, Royal Victoria Hospital Site, Grosvenor Road, Belfast, BT12 6BJ, Northern Ireland, United Kingdom; Tel: +0044 (0) 2890971606; E-mail: c.treanor@qub.ac.uk

\section{Acknowledgements}

The authors wish to acknowledge Richard Fallis, the information specialist in the Medical Library, Queen's University Belfast. 


\begin{abstract}
Purpose

To systematically review and synthesise randomised controlled trials investigating the effectiveness of prehabilitation compared to usual care for newly diagnosed, adult-onset cancer patients.
\end{abstract}

Methods

MEDLINE, EMBASE, PsycINFO, CINAHL and SSCI were searched up to April 2017. Studies were included if disease-, treatment-related, patient-reported and health service utilisation outcomes were assessed. Two reviewers independently reviewed and appraised the risk of bias of each study.

Results

Eighteen studies were included. Interventions comprised one or more of the following components: psychological support, education and exercise. Meta-analyses found that pelvic floor muscle training (PFMT) significantly increased odds of continence at three-months (OR=3.29, 95\%CI=1.57-6.91), but did not significantly reduce daily pad use at six-months post-surgery $(\mathrm{MD}=-0.96,95 \% \mathrm{CI}=-2.04-0.12)$ for prostate cancer patients. Although quality-of-life improved due to PFMT, functional ability or distress did not. Further meta-analyses indicated that pre-surgical exercise significantly reduced length of hospital stay $(\mathrm{MD}=-4.18$, $95 \% \mathrm{CI}=-5.43--2.93)$ and significantly lowered odds of post-surgery complications $(\mathrm{OR}=0.25,95 \% \mathrm{CI}=0.10$ 0.66) for lung cancer patients. Psychology-based prehabilitation significantly improved mood, physical wellbeing and immune function for prostate cancer patients, and improved fatigue and psychological outcomes and a trend for better quality-of-life among breast cancer patients. Risk of bias was high for most studies.

\title{
Conclusions
}

Prehabilitation appears to benefit cancer patients. Rigorous trials are needed to investigate the effectiveness of prehabilitation among other cancer sites and other related effects. The cost-effectiveness of prehabilitation remains unanswered.

Implications for cancer survivors

Providing interventions earlier in the care pathway may lead to better outcomes for patients during survivorship.

Keywords: cancer, prehabilitation, systematic review, meta-analysis 


\section{Introduction}

Cancer is a major global health problem, with most recent estimates reporting 14.1 million new cancer cases worldwide in 2012 [1]. Although treatments are becoming more effective, resulting in decreased mortality from cancer, cancer and cancer treatment remain challenging for patients and their families. Recently diagnosed cancer patients experience a range of emotional responses and uncertainty about what to expect from treatment $[2,3]$, and are at risk of developing physical, psychological and social 'late consequences' as a result of cancer and its treatment [4]. These changes have a detrimental effect on patients' lives in domains such as returning to work, family relationships, activities of daily living and, generally, quality-of-life (QoL) [4-6]. It is important to investigate strategies that might maximise support for patients during the pre-treatment period given the increasing number of individuals living for longer with cancer and consequentially with its effects.

The period between cancer diagnosis and treatment presents a unique opportunity to support and prepare patients for treatment. This pre-treatment or prehabilitation phase provides cancer patients with a programmatic way, potentially, to prepare physically and psychologically for cancer treatment and the challenges of 'survivorship'. More specifically, there may be significant beneficial effects for patients in terms of developing coping resources and skills and improving recovery from cancer treatment. In turn, the benefits of a prehabilitation programme may lead to an appropriate reduction in use of health services and a maximisation of post-treatment physical and psychological outcomes [7,8]. This systematic review aims to assess the effectiveness of cancer prehabilitation interventions versus standard care on physical, psychological and health service utilisation outcomes.

\section{Methods}

This review was conducted in accordance with Cochrane Collaboration methods [9] and reported according to the Preferred Reporting Items for Systematic Reviews and Meta-analysis (PRISMA) checklist [10]. The following sources were searched: EMBASE, MEDLINE, PsycINFO, Cumulative Index of Nursing and Allied Health Literature (CINAHL) and Social Sciences Citation Index (SSCI) up to $3^{\text {rd }}$ April 2017 (see Supplementary Figure $\mathrm{i}$ for search strategy). The eligibility criteria were applied independently to the titles, abstracts and subsequently full texts of each paper by two reviewers (CT and TK). A third reviewer (MD) was available in the event that no consensus was reached. The citation lists of identified reviews and included studies were also searched and citations assessed for eligibility. The review is registered on PROSPERO (ID: CRD42016036072). 


\section{Eligibility criteria}

Randomised controlled trials (RCTs) which compared one or more prehabilitation interventions to standard or usual care were included in the review. Prehabilitation interventions were defined according to the following criteria: baseline assessment of patients; initiation prior to acute treatment with curative treatment (chemotherapy, radiotherapy or surgery) for newly diagnosed, adult-onset cancer patients and; aimed to have an impact on physical-, or psychological- health and health service utilisation outcomes. Interventions which continued during or after treatment were included [11]. The following intervention types were excluded: pharmacological interventions delivered in isolation or in combination with non-pharmacological interventions and preoperative procedures occurring within hours of treatment. Studies including patients with benign tumours were included only if outcome data were reported separately for benign and malignant tumour groups. Study populations with secondary or metastatic cancers were excluded.

\section{Data extraction, synthesis and risk of bias assessment}

Data were extracted independently by two reviewers (CT and TK) (see Supplementary table 1 for study characteristics). Data were examined for suitability to be included in a meta-analysis. Some data could be pooled statistically using Cochrane Collaboration's Review Manager (RevMan) version 5.3 using random effects models. Heterogeneity was assessed using the $\mathrm{I}^{2}$ statistic. The remaining data were synthesised narratively. The following aspects of each study were assessed for risk of bias using the Cochrane Collaboration risk of bias tool [9]: random sequence generation, allocation concealment, participant and personnel blinding, outcome blinding, incomplete outcome assessment and selective outcome reporting. Risk of bias was assessed independently by two reviewers (CT and TK); a third reviewer (MD) was available to settle any non-consensus. Risk of bias for each study was categorised as follows: (i) low if all criteria were scored as low risk of bias, (ii) moderate if one or two criteria were scored as unclear or high risk of bias and (iii) high if more than two criteria were scored at unclear or high risk of bias. We considered studies of all levels of methodological quality and decided to include less robust studies because of the sparseness of the literature and in order to maximise potential lessons from the review. The degree to which methodological weaknesses affected or diluted any lessons or arguments is highlighted in the Discussion.

\section{Results}

A total of 18 studies were included in the review (see supplementary Figure ii for study eligibility). One paper reported the findings of two intervention studies [12] and two papers reported different outcomes regarding the 
same intervention $[13,14]$. Studies were conducted in the USA $(n=8)$, Italy $(n=3)$, the UK $(n=1)$, the Netherlands $(n=1)$, Turkey $(n=1)$, Australia $(n=1)$, Switzerland $(n=1)$, China $(n=1)$ and Denmark $(n=1)$. A total of 1381 patients were enrolled across the studies including individuals with lung cancer $(n=7)$, prostate cancer $(n=5)$, breast cancer $(n=4)$ bladder cancer $(n=1)$ and multiple cancer sites $(n=1)$.

The majority of interventions were implemented prior to surgery; one study was implemented before radiotherapy [15]. Interventions were implemented one to two weeks $(n=9)$, one to five days $(n=2)$, one month $(n=2)$, three weeks $(n=1)$ and the day preceding treatment $(n=1)$. Three studies did not specify the time-frame of intervention implementation prior to treatment [16-18]. Standard care varied according to individual institutions whereby verbal or written instructions were given [15,19-21], physical activity was delivered in the rehabilitation setting post-treatment [22,23], generic risk management and active mobilisation was given [18] or fast-track surgery was implemented [24]. Five studies delivered interventions in the pre-treatment period only $[12,17,18,25-27]$; the remaining studies continued the interventions during or after treatment. In total, twentytwo interventions were compared to a usual care control group. The interventions included pelvic floor muscle training (PFMT) with biofeedback $(n=4)$, psychological interventions $(n=4)$, education $(n=4)$, pulmonary rehabilitation $(\mathrm{n}=4)$, physical activity $(\mathrm{n}=3)$, gown $(\mathrm{n}=1)$ and combined gown and education intervention $(\mathrm{n}=1)$. Two psychological interventions were compared to 'attentional' control groups $[13,14,23]$. The intensity of intervention delivery varied between the studies including: two contacts $(n=4)$, one single contact $(n=3)$, ten contacts $(n=2), 20$ contacts $(n=2)$, six contacts $(n=1), 15$ contacts $(n=1), 14$ contacts $(n=1)$ four contacts $(n=1)$, three sessions $(\mathrm{n}=1)$, two to three contacts per week $(\mathrm{n}=1)$ and six sets of daily exercises over a 7 -day period $(n=1)$.

\section{Risk of bias}

Fourteen studies were scored as having high risk of bias, two studies were scored as having moderate risk of bias and a further two studies were scored as having low risk of bias (see Supplementary Figure iii). There was evidence of selective or incomplete outcome reporting within the studies. The randomisation process, allocation concealment and blinding processes were poorly described in many of the studies.

\section{Prostate cancer}

\section{PFMT versus usual care}


Four studies investigated the effects of PFMT versus usual care on continence-related outcomes [16,19,21,28], QoL [21,28], lifestyle and functional ability [21] and satisfaction [28]. Studies were rated as having high $[16,21]$, moderate [19] and low [28] risk of bias.

A meta-analysis of three studies (of low, moderate and high risk of bias ratings, respectively) $[16,19,28]$ indicated that patients who undertook PFMT were over three times significantly more likely than patients who received usual care to recover urinary continence at three-months post-surgery $(\mathrm{OR}=3.29,95 \% \mathrm{CI}=1.57-6.91$, $\mathrm{I}^{2}=15 \%$ ) (see Figure i). The following continence-related results emanate from individual studies which could not be combined in a meta-analysis unless stated otherwise. For example, at one-month post-treatment there was a significant benefit on recovery of continence for patients undertaking PFMT compared to controls (Intervention (IV): 44.1\% $(n=26)$ versus Control (C): 20.3\% $(n=12), p=0.018)$ [28]. Compared to controls, patients who underwent PFMT had significantly shorter median time to continence recovery (IV: 12 weeks versus C: 16 weeks, $\mathrm{p}<0.05)$ [16]. At one- and six-months post-treatment, there was a significant benefit of PFMT on the proportion of patients who achieved continence compared to the control group (6 versus 0 , $\mathrm{p}=0.02 ; 10$ versus $1, \mathrm{p}=0.002$, respectively) [19]. A further study found that men who underwent PFMT were significantly quicker to recover continence at six-months post-surgery compared to controls (Kaplan Meier log rank: $\mathrm{p}=0.04)$. Moreover, at six-months there was $20.03 \%$ difference in proportion of men who recovered continence in the intervention and control groups, favouring the intervention group [21].

Findings from individual studies report that at one-month and three-months post-treatment, the PFMT group had around a $60 \%$ reduced odds of being incontinent compared to the control group $(\mathrm{OR}=0.41,95 \% \mathrm{CI}=0.20-0.84$, $\mathrm{p}=0.016$ and $\mathrm{OR}=0.38,95 \% \mathrm{CI}=0.18-0.79$, respectively) [28]. The control group experienced significantly more incontinence episodes compared to the intervention group at three-months (IV: 3.84 versus C: $13.06, p=0.01$ ) and six-months (IV: 2.72 versus C: 13.06, p=0.005), but not at one-month post-treatment [19]. Participants in one study underwent a 24-hour pad weight test as an indication of incontinence, at both one-month and threemonths follow-up significantly fewer participants who underwent PFMT had a pad $>150$ grams compared to controls (IV: $25.4 \%$ vs C: $33.9 \%, p=0.04$ and IV: 16.9 vs C: $32.2, p=0.033$, respectively) [28].

Two studies (with a moderate and a high risk of bias rating, respectively) [19,21] were combined in a metaanalysis and this analysis pointed to a trend for improvement among patients who underwent PFMT compared to usual care regarding daily pad use six-months post-surgery $\left(\mathrm{MD}=-0.96,95 \% \mathrm{CI}=-2.04-0.12, \mathrm{I}^{2}=90 \%\right)(\mathrm{see}$ Figure ii). One study found that at one-year post-surgery, one patient in each group used between one and three 
pads daily; three patients $(15.8 \%)$ in the control group and two patients $(10.5 \%)$ used more than three pads daily $[16]$.

At six-months post-intervention, a significantly lower proportion of participants in the intervention group compared to the control group had severe/continual leakage ( $\mathrm{IV}=5.9 \%$ versus $\mathrm{C}=19.6 \%, \mathrm{p}=0.04$ ). Moreover, the intervention group spent significantly more days without leaks ( $I V=72.6$ versus $C=54.2, p=0.04)$ [21]. There were significant between-group differences (favouring the intervention group) in survey-assessed overactive bladder and urinary function at three- (IV:10.12 versus C:13.19, p=0.04 and IV:403.81 versus C:272.44, $p=0.006$, respectively), six-months (IV:9.06 versus $C: 12.62, p=0.01$ and IV:422.50 versus $C: 274.25, p=0.003$, respectively), but not one-month post-treatment [19]. Compared to patients who underwent PFMT, patients who received usual care were significantly more likely to experience episodes of urine loss due to: coughing ( $\mathrm{n}=11$ vs. $n=24, p=0.003)$, sneezing ( $n=13$ vs. $n=23, p=0.02)$ and, getting up from a lying down position $(n=7$ vs. $n=15$, $\mathrm{p}=0.04)$ [21]. There were no significant between-group differences on other reasons for urine loss (e.g. due to lifting or walking) [21].

A meaningful meta-analysis could not be conducted for the remaining outcomes for PFMT versus usual care due to heterogeneity. So, findings from individual studies are reported in narrative form. There were no significant between-group differences in proportion of patients who returned to work or resumed usual activities or in lifestyle factors, distress or QoL [21]. Continence-related QoL was significantly improved in the intervention compared to the control group at one-month (IV: 14.6 versus $\mathrm{C}: 18.3, \mathrm{p}=0.002$ ) and three-months (IV: 8.1 versus C: $12.2, \mathrm{p}=0.002$ ) post-treatment [28]. Patterns of continence-related QoL varied according to whether patients were completely dry, occasionally incontinent or incontinent. The significant between-group difference favouring the intervention group remained at three-months post-treatment for patients who were dry after two months (IV: 3.8 versus C: 5.5, $\mathrm{p}<0.001$ ). A significant benefit in incontinence-related QoL for the intervention group compared to the control group remained at one-month (IV: 14.5 versus $\mathrm{C}: 16.2, \mathrm{p}=0.006)$ and three-months (IV: 8.5 versus $\mathrm{C}: 10.5, \mathrm{p}=0.014$ ) for patients who were occasionally incontinent. There was a significant improvement in incontinence-related QoL (favouring the intervention group) at one-month (IV: 21.9 versus $\mathrm{C}: 25.9, \mathrm{p}<=0.001$ ), but not three-months (IV: 22.2 vs $\mathrm{C}: 22.3, \mathrm{p}=0.886$ ) post-treatment among patients who were incontinent [28].

Seventy-five percent of patients in the PFMT intervention reported feeling extremely satisfied with the intervention [28]. 


\section{Psychological intervention versus usual care}

One study (reported in two reports) investigated the effects of stress management training (SMT) versus supportive attention (SA) and standard care on psychological [13], QoL [13], biological [14], health service utilisation [13] and post-operative complication [13] outcomes among prostate cancer patients. This study was rated as having high risk of bias [13,14]. The effect of SMT versus SA and standard care on mood disturbance measured using the Profile of Mood States (POMS) at one-week pre, and morning of-, surgery was assessed using generalised linear mixed models controlling for several factors. A significant main effect for group $(\mathrm{p}=0.02)$ and time $(\mathrm{p}=0.04)$ was found with the SMT group having lower levels of mood disturbance over time and compared to the standard care group, There was an absence of a group by time interaction for mood disturbance [14]. The significant between-group differences were not sustained in the long-term from six-week to 12-month follow-up. Trial findings indicate that there were no significant between- or within-group differences with regards to scores on the Impact of Events Scale. Only the Physical Component Summary (PCS) score of the SF-36 demonstrated a significant between-group difference $(p<0.004)$ and significant changes over time at six weeks, six months and 12 months $(\mathrm{p}<0.02)$, with the SMT group reporting better physical well-being than the standard care group. A significant group by time interaction was absent for PCS scores. Significant changes over the course of the intervention were observed for various domains of the prostate cancer-specific QoL including urinary -function $(\mathrm{p}<0.0001)$, -limitation $(\mathrm{p}<0.0001)$, -bother $(\mathrm{p}<0.0001)$, sexual function $(p<0.0001)$ and cancer worry $(p<0.004)$. A decline in scores for each domain was observed from baseline to six -weeks and -months post-surgery and an increase by 12-months post-surgery. There were no significant group by time interactions for prostate cancer-specific QoL [13].

Immunological markers were collected at baseline, one-month prior to surgery and 48-hours post-surgery. Natural Killer Cell Cytotoxicity (NKCC) levels were significantly higher in the SMT group compared to both SA and standard care groups (levels in both groups decreased, whilst levels in the SMT group increased) at the 80:1 peripheral blood mononuclear cells $(\mathrm{PBMC})$ effector $(\mathrm{E})$ and K562 cell line target $(\mathrm{T})$ ratio $(\mathrm{p}=0.04)$ and a trend for significance for the 40:1 E:T ( $\mathrm{p}=0.06)$. Pro-inflammatory markers, Interleukin (IL)-12p70, IL-1 beta $(\beta)$ and Tumour Necrosis Factor alpha $(\mathrm{TNF}-\alpha)$ were significantly higher in the SMT group compared to the SA group ( $\mathrm{p}=0.028, \mathrm{p}=0.018$ and $\mathrm{p}=0.05$, respectively). Only IL- $1 \beta$ was significantly higher $(\mathrm{p}=0.05)$ in the SMT group compared to the standard care group. There was a trend for higher IL-8 levels $(\mathrm{p}=0.06)$ in the SMT group compared to the other groups. There were no between-group differences between the SA and standard care 
groups [14]. There was an absence of between-group differences in surgery-related problems and other complications and frequency of hospital admissions until the end of follow-up at 12-months [13].

\section{Lung cancer}

\section{Pre-surgical exercise versus usual care}

Seven studies investigated the effects of pre-surgical exercise compared to usual care on health service utilisation $[12,17,18,22]$, post-operative complications $[12,17,18,22]$, cardiopulmonary $[12,17,18,22,25]$, QoL [17], and psychological [26] outcomes among lung cancer patients. Each study was rated as having a high risk of bias.

Findings from three studies $[12,17,22]$ were combined in a meta-analysis which indicated a significant benefit for pre-surgical pulmonary exercise versus usual care on total number of days spent in hospital among lung cancer patients $\left(\mathrm{MD}=-4.18,95 \% \mathrm{CI}=-5.43--2.93, \mathrm{I}^{2}=0 \%\right)$ (see Figure iii). Findings from individual studies on other health service utilisation outcomes reported a reduction in the number of days spent in hospital postoperation (pulmonary prehabilitation [mean=4.4] compared to the usual care group [mean=6.9], $\mathrm{p}=0.010$ ) [17]. Furthermore, high intensity interval training (HIIT) led to a significant reduction in hours spent in recovery from anaesthesia (median=25 versus 17, $\mathrm{p}<0001$ ) compared to patients who did not participate in HIIT, however, there was no significant between-group difference for days spent in hospital [18]. There were no significant between-group differences for mean number of intensive care unit hours or mean ventilation hours as a result of pulmonary exercise [12]. There were significantly fewer lung cancer patients in the preoperative exercise group who experienced prolonged chest intubation $(n=1$ vs $n=5, p=0.03)$ and had significantly fewer mean number of days with a chest tube $(4.3(\mathrm{sd}=2.1)$ vs $8.8(\mathrm{sd}=5.30), \mathrm{p}=0.04)$ compared to patients who received usual care [12].

A meta-analysis combining findings from three studies [12,17,22] found benefits for pre-surgical exercise compared to usual care for post-operative complications among lung cancer patients $(\mathrm{OR}=0.25,95 \% \mathrm{CI}=0.10$ $0.66, \mathrm{I}^{2}=0 \%$ ) (see Figure iv). Results from individual studies that could not be included in the meta-analysis found that the incidence of respiratory complications was lower among patients who participated in HIIT compared to usual care. There were no significant between-group differences in cardiovascular-, surgicalcomplications, wound infection or renal dysfunction as a result of HIIT [18]. Regarding specific complications, a control group in one study [22] experienced fever $(n=2)$, atelectasis $(n=1)$, pneumonia $(n=1)$ and haemorrhagic drainage $(n=1)$, whereas fever was experienced by one patient in the intervention group. In a further study [17], 
grade $1(n=15$ versus $n=16)$, grade $2(n=4$ versus $n=8)$, grade $3(n=2$ versus $n=4)$, grade $4(n=0$ versus $n=1)$ and grade 5 ( $n=0$ versus $n=1$ ) complications were experienced by the intervention and control groups, respectively. There were no significant between-group differences in the proportion of patients with respiratory failure, pneumonia or atelectasis in an additional study [12].

Findings could not be combined in a meta-analysis for cardiopulmonary outcomes so the following results refer to individual studies. In one study, from pre- to post-intervention, the intervention group experienced significant improvements in forced expiratory volume $\left(\mathrm{FEV}_{1}\right)(\mathrm{p}<0.001)$, forced volume capacity $(\mathrm{FVC})(\mathrm{p}=0003)$, carbon monoxide diffusion capacity $(\mathrm{DLCO})(\mathrm{p}<0.001)$, partial arterial oxygen pressure $\left(\mathrm{PaO}_{2}\right)(\mathrm{p}<0001)$ and a significant decrease in partial arterial carbon dioxide pressure $\left(\mathrm{PaCO}_{2}\right)(\mathrm{p}=0.004)$ [22]. A further study of elderly patients found significant between-group differences in peak expiratory flow, but not DLCO, FEV 1 and FVC post-intervention [17]. A study investigating the effects of HIIT found improvements in peak oxygen volume $\left(\mathrm{VO}_{2}\right)(\mathrm{p}=0.004)$ and peak work rate $(\mathrm{p}=0.021)$, but no other differences in other cardiopulmonary measures compared to the usual care group were found [18]. Participants with lung cancer and comorbid chronic obstructive pulmonary disease (COPD) who received a pre-operative pulmonary rehabilitation programme (versus usual care) had significantly increased peak volume oxygen $\left(\mathrm{VO}_{2}\right)$ levels on the day of surgery $(p<0.0001)$ and 60 days post-surgery $(p<0.005)$. However, significant between-group differences in pulmonary function $\left(\mathrm{FEV}_{1}\right)$ were not observed at any time-point [25]. There was a significant beneficial effect of exercise on total heart rate $(p=0.04)$ [22], walking distance $((p<0.001)[22],(p=0.001)[18],(p=0.029)[17])$, walking speed $(p<0.001)[22]$ and walking duration $(p<0.001)[22]$ compared to usual care. In a further study, there were no significant between-group differences in the shuttle walk test [12].

An exercise intervention combining respiratory, arm, leg, walking and stair climbing exercises was compared to a usual care group on hope and empowerment measures. There were no significant between- or within- group differences in hope over the course of the trial. A significant benefit in power for the intervention group was found from pre- to post-intervention was found $(\mathrm{t}=-2.68, \mathrm{p}=0.01)$, but not from post-intervention to four to six days post-surgery $(\mathrm{t}=-1.89, \mathrm{p}=0.07)$. However, a significant increase in power from pre-intervention to four to six days post-surgery $(t=-3.73, p=0.001)$ was observed for the exercise group. There was a significant decrease in power from pre- to post-intervention $(\mathrm{t}=2.72, \mathrm{p}<0.01)$ for the control group, and power decreased (albeit not significantly so) from post-intervention to four to six days post-surgery $(t=-0.29, p=0.78)$ [26]. ANOVA models indicated that there was a significant between-group difference post-intervention $(F(1,143)=4.77, p=0.03)$ and four to six days post-intervention $(\mathrm{F}(1,143)=11.13, \mathrm{p}<0.01)$ favouring a beneficial improvement in power for 
the exercise group. Bonferroni-corrected t-tests indicated significant increase in power from pre- to postintervention $(\mathrm{t}=-2.68, \mathrm{p}=0.01)$ and pre-intervention to shortly after surgery $(\mathrm{t}=-2.68, \mathrm{p}=0.01)$ for the intervention group. This improvement was not significant from post-intervention to shortly after surgery. A different pattern of power emerged for participants in the control group whereby power decreased over time but was only significant from pre- to post-intervention $(\mathrm{t}=2.72, \mathrm{p}=0.009)$ [26].

One study measured the effect of pulmonary prehabilitation on QoL outcomes with no benefits for patients [17].

One trial was terminated early due to slow recruitment (under-powered sample), interim analysis found there were no significant between-group differences in any outcome. The trial was deemed to be infeasible as patients were reluctant to delay treatment to participate in the trial [12].

\section{Breast cancer}

\section{Psychosocial intervention versus usual care}

Three studies investigated the effectiveness of psychosocial interventions compared to usual care on a range of somatic [23,29], biological markers [27] and psychological outcomes [23,27,29]. Each study was rated as having high risk of bias.

In one study, breast cancer patients scheduled to receive surgery were allocated to a psychotherapeutic intervention group, two attentional control groups or a usual care group. However, the investigation of intervention effectiveness was limited because the usual care group was not assessed on outcome measures preoperation. An intervention effect on recovery- or physiological indices was not observed [23]. A study investigated the effects of a psychosocial intervention (including psychoeducation about stress, problem-solving and relaxation skills and supportive discussion) compared to usual care on immunological markers and psychological variables. The study found no significant interaction or main effect of time or group allocation on NKCC levels. However, there was a significant group by time allocation interferon gamma (IFN $\gamma$ ) indicating maintenance of IFN $\gamma$ levels among patients in the intervention group and a decrease of IFN $\gamma$ levels among the control group. A significant between-group difference was observed pre-intervention and, therefore, an ANCOVA model was undertaken to control for pre-intervention IFN $\gamma$ levels. The ANCOVA model did not indicate or retain a significant group by time interaction [27].

Intervention and attentional control groups observed significant benefits for distress-related body image compared to controls at three months and one-year post-surgery $(\mathrm{F}=12.3, \mathrm{p}=0.0006)$. The control group 
(compared to participants in any of the other groups) had significantly higher levels of distress $(\mathrm{p}=0.024)$, anxiety and depression $(\mathrm{p}=0.005)$ as assessed by questionnaire at three-months post-surgery. One-year postsurgery, significantly higher levels of distress related to loss of breast ( $\mathrm{p}=0.004)$, partner's response and total worries were found for controls compared to intervention participants. The control group was less likely to report fighting spirit as a coping approach and were more likely to have caseness for anxiety or depression (25\% versus $10 \%)$ [23]. The interest subscale $(\mathrm{F}=20.1, \mathrm{df}=1.24, \mathrm{p}<0.0003)$ and enjoyment subscale of the Differential Emotions Scale (DES) $(\mathrm{F}=15.4, \mathrm{df}=1.24, \mathrm{p}<0.0003)$ increased over time from pre- to post-intervention, whereas the sadness subscale $(\mathrm{F}=7.2, \mathrm{df}=1.24, \mathrm{p}=0.01)$ decreased over time for the intervention group. A significant group by time interaction was found for DES disgust whereby disgust increased and decreased over time for the control and, intervention and attentional control groups, respectively. There were no significant main effects for the remaining eight emotions measured by this scale. A significant main effect from pre- to post-intervention for the Life Orientation Test (LOT) measuring optimism was found $(\mathrm{F}=4.6, \mathrm{df}=1.27, \mathrm{p}<0.05)$. There were no significant benefits of the intervention versus control group in optimism, depression or Impact of Events scale (IES) [27].

Breast cancer patients were randomised to a SMT group or usual care. Significant between-group differences at five-days post-surgery were observed for depression and two and five-days post-surgery for fatigue whereby the SMT group reported a decrease in both outcomes. There were no significant between-group differences in anxiety, QoL, perceived control or sleep. Patients in SMT group experienced an improvement in QoL at days two and 30 post-surgery and both groups experienced a reduction in anxiety over time [29].

\section{Education and Papilla gown versus usual care}

One study (rated as having high risk of bias) investigated education, Papilla gown and combined education and Papilla gown interventions with usual care of a standard hospital gown on activity levels, cancer knowledge, body image, lymphedema and comfort levels for breast cancer patients [20].

Activity levels increased over time for patients $(\mathrm{p}<0.001)$; patients who wore the Papilla gown compared to those who did not had significantly higher levels of activity (Papilla gown mean=4.88 versus Usual care mean $=4.59, \mathrm{p}=0.026$ ). The combined education and gown intervention did not lead to any significant main effects or interactions for activity levels. One-week post-operation, use of the Papilla gown demonstrated a beneficial effect on activity levels compared to participants who did not wear the gown (Papilla gown mean $=4.50$ versus usual care mean $=4.21, \mathrm{p}=0.039)$. There was a significant beneficial effect $(\mathrm{p}<0.001)$ of 
education (mean $=0.34)$ compared to no education (mean $=0.11$ ) on cancer knowledge. A significant interaction was found between education and wearing the Papilla gown $(\mathrm{p}=0.037)$. One-week and one-month postoperation, participants in the Papilla gown group had a significant improvement in breast cancer knowledge compared to participants who were not in the Papilla gown group (Papilla gown mean $=0.27$ versus Usual care mean $=0.20, \mathrm{p}<0.001$ ). Similarly, receipt of education versus no education had a significant improvement on breast cancer knowledge (education mean $=0.36$ versus no education mean $=0.11, \mathrm{p}<0.001$ ). Education only at six months post-operation was significantly associated with improvement in breast cancer knowledge compared to no education receipt $(\mathrm{p}=0.014)$. Neither the Papilla gown nor the education receipt had a significant effect on body image for breast cancer patients.

Patients who wore the Papilla gown experienced significantly more comfort compared to the usual care group (Papilla gown mean $=4.41$ versus Usual care mean $=3.00, \mathrm{p}<0.001$ ). There was a beneficial effect of wearing the Papilla Gown compared to the standard gown at one-week (Papilla gown mean $=4.46$ versus Usual care mean $=2.53, \mathrm{p}<0.001)$ and an increase in comfort six-months post-operation $(\mathrm{p}=0.006)$ for the Papilla gown group. There was a significantly lower incidence of lymphedema among patients who wore the Papilla gown compared to those who did not $(\mathrm{p}=0.0097)$ from baseline to post-intervention. Comfort levels significantly improved over time from $\mathrm{T} 1$ to $\mathrm{T} 2$ for patients $(\mathrm{p}=0.0004)$ [20].

\section{Bladder cancer}

One study (rated as having moderate risk of bias) compared a standardised fast-track pathway to an intensive pre-operative exercise intervention which continued post-operatively for patients treated with radical cystectomy. The study found no significant benefits for patients in terms of length of hospital stay $(\mathrm{p}=0.68)$, complication incidents $(\mathrm{p}=0.47)$, severity of complications $(\mathrm{p}=0.64)$, hospital readmissions $(\mathrm{p}=0.49)$, mortality $(p=0.84)$, bowel function restoration $(p>0.05)$, energy intake $(0.60)$ or protein intake $(p=0.29)$ [24].

\section{Multiple cancer sites}

One study (rated as having low risk of bias) investigated the effects of a psychoeducational, cancer-related fatigue intervention trial (CAN-FIT) guided by the Health Belief Model to encourage self-care strategies to reduce fatigue among cancer patients [15].

The intervention was delivered pre-radiotherapy only, post-radiotherapy only or pre- and post- radiotherapy and compared to usual care. There were no differences in fatigue, QoL, depression, anxiety, physical activity levels 
or occupational activities between the CAN-FIT and usual care groups. There were some group differences depending on when the CAN-FIT intervention was delivered. Between baseline and post-radiotherapy assessments there was a significant increase in moderate activity levels $(\beta=1.4,95 \% \mathrm{CI}=0.53-2.26)$, vigorous activity levels $(\beta=1.05,95 \% \mathrm{CI}=0.24-1.86)$ and a significant decrease in QoL utility scores $(\beta=-0.11,95 \% \mathrm{CI}=$ 0.22-0.00) among participants who received CAN-FIT pre-radiotherapy. A significant increase in vigorous activity levels $(\beta=1.24,95 \% \mathrm{CI}=0.44-2.03)$ and a significant decrease in hours of paid work $(\beta=-0.72,95 \% \mathrm{CI}=$ -1.44- -0.04) was found for participants who received CAN-FIT pre-radiotherapy compared to those who did not receive CAN-FIT pre-radiotherapy from baseline to six-weeks follow-up. Patients who did not receive CANFIT post-radiotherapy experienced a beneficial effect on mental fatigue $(\beta=-1.66,95 \% \mathrm{CI}=-3.21--0.10)$ and QoL utility scores $(\beta=0.10,95 \% \mathrm{CI}=0.00--0.20)$. Moreover, patients who received CAN-FIT post-radiotherapy walked significantly more per week $(\beta=5.82,95 \% \mathrm{CI}=0.07-11.56)$ and were significantly more likely to participate in unpaid work $(\beta=561.79,95 \% \mathrm{CI}=51.21-1072.37)[15]$.

In terms of adherence to the intervention, $55 \%$ (range $=48-66 \%)$ fully adhered to the intervention, $59 \%$ (range= $52-66 \%$ ) completed $75 \%$ of the intervention, $68 \%$ (range $=60-74 \%$ ) adhered to half of the intervention and $14 \%$ (range $=8-18 \%$ ) adhered to $30 \%$ of the intervention. Psychological factors (e.g. anxiety), somatic factors (e.g. pain, fatigue) and acute side effects from procedures such as discomfort from catheterisation were reported as reasons for not fully adhering to the intervention [15].

\section{Conclusion}

Clearly, there is a need to consider ways in which to maximise treatment effectiveness, recovery and survival in the face of the increasing number of individuals diagnosed with cancer and the subsequent demands and pressure that this places on health care systems and services. This review demonstrates that prehabilitation may be one way in which to meet these challenges as it appears, overall, to have several benefits for patients. For example, PFMT improved urinary continence outcomes and QoL for prostate cancer patients though there were no improvements in functional ability or distress. Psychological-based prehabilitation interventions led to improved mood, better physical wellbeing and immune function for prostate cancer patients and lower distress, depression, anxiety, fatigue, emotional states and increased optimism and fighting spirit, with a trend for better QoL among breast cancer patients. Preoperative exercise had several benefits for lung cancer patients in terms of pulmonary function, functional ability, health service utilisation and experience of treatment-related complications, even for patients with co-morbid illnesses. Education interventions also demonstrated benefits 
for patients. Although the effectiveness of prehabilitation on QoL was not significant, trends in QoL improvements were observed. It may be that interventions were not of sufficient intensity to lead to clear sustained improvements in QoL and 'booster' sessions may be required. Perhaps, ceiling effects regarding QoL may be present at this critical time-point given the nature of treatment that is received and related acute side effects likely to be experienced by patients.

Few studies have addressed the effectiveness of delivering interventions prior, as opposed, to following cancer treatments. The CAN-FIT study did not find any additional benefits of implementing a psychoeducation intervention pre-radiotherapy compared to post-radiotherapy [15]. However, enhanced benefits relating to urinary incontinence were found for prehabilitative PFMT [19]. Other studies not included in the review (because they did not meet one or more eligibility criteria) found improved outcomes for patients in the prehabilitation versus rehabilitation period. For example, combined interventions for patients with colorectal(exercise, nutrition and counselling) [30] or lung- cancer (exercise and nutrition) [31]. Preoperative exercise for lung cancer patients was associated with a number of benefits for patients [32]. Prehabilitative versus rehabilitative swallowing- or respiratory therapy led to significant benefits for patients with head and neck cancers [33-35]. There appears to be a potentially significant advantage to implementing prehabilitation rather than rehabilitation interventions for cancer patients.

Most of the studies in the review did not investigate the influence of prehabilitation on recurrence or survival outcomes. Physical activity and exercise are beneficial in terms of the primary and secondary prevention of cancer as well as cancer-related and all-cause mortality [36]. Future research should investigate the degree to which prehabilitation exercise programmes as an adjunct to traditional cancer therapies present an advantage for patients in terms of survival and recurrence.

Usually, there is a short window of opportunity for prehabilitation to be implemented prior to treatment. Timeappropriate implementation coupled with patient anxiety and uncertainty about what to expect from imminent treatment and its outcomes is challenging for service delivery staff, patients and families. According to results of the review, prehabilitation appears to be acceptable to patients and feasible to implement with good levels of adherence supported by studies within the review [15,18,29]. This finding is further supported by the wider research literature [37]. Even an intensive prehabilitation exercise programme compared to home-based exercise was found to have good levels of adherence [38], as long as prehabilitation did not interfere with receipt of cancer treatment [12]. Other studies have found that exercise in the prehabilitation period was feasible for 
sedentary individuals [39]. The results from a single case report hinted that prehabilitation may be beneficial for elderly patients [40] which in turn supported the findings by Lai et al. (2017) [17].

\section{Study limitations}

This review was guided by Cochrane Collaboration methods and the PRISMA statement and has various strengths. Paper selection, risk of bias assessment, data extraction were undertaken independently and the search included a range of multi-disciplinary bibliographic databases. However, the review did not include searches of the grey literature, non-English language publications or non-randomised studies which are potential limitations. Moreover, various included studies were rated as having high risk of bias or had a small number of trial participants. We decided not to exclude studies of low methodological quality or with potentially underpowered samples so as to give a comprehensive overview of the nature and type of prehabilitation studies within cancer care. Therefore, this fact should be borne in mind when considering the results of the review.

\section{Clinical implications}

Global policy priorities recognise the need to plan and provide long-term support for the increasing population of cancer survivors. In various countries, clinical guidelines have been developed to identify and address the psychosocial needs of cancer survivors; and there is a concentrated drive to deliver high quality cancer survivorship care and to intervene appropriately at the point of diagnosis [41]. Whilst there is movement towards the implementation of evidence-based interventions in the period post-cancer treatment, there is a need to research and consider recommending the implementation of interventions in the prehabilitation period, as part of usual care. The review findings presented here indicate that prehabilitation may be potentially effective though further research is required including studies of cost-effectiveness and scope for appropriate reductions in health service utilisation, less post-operative complications and improvements in QoL. Studies from related fields point to the likelihood of the cost-effectiveness of prehabilitation. For example, one study calculated incremental cost-effectiveness ratios (ICERs) for pre-treatment swallowing therapy and found that it was costeffective compared to swallowing therapy in the rehabilitative period [42].

This is the first comprehensive systematic review to the best of our knowledge that has addressed the full range of prehabilitation interventions among cancer patients irrespective of cancer site. Mindful of the results of the risk of bias analysis, there appear to be benefits to delivering interventions to cancer patients during the prehabilitation period in terms of reduced health service utilisation, reduced frequency and severity of treatmentrelated effects and other important patient outcomes. However, there is a need for rigorous trials to examine and 
test prehabilitation and its cost-effectiveness particularly among cancer sites other than breast, prostate or lung cancer and to consider whether interventions delivered early in the care pathway combat cancer-related effects such as pain, sleep and cognitive changes.

\section{Compliance with Ethical Standards:}

Funding: This study did not receive any financial support.

Conflict of Interest: Author Charlene Treanor declares that she has no conflict of interest. Author Thinzar Kyaw declares that she has no conflict of interest. Author Michael Donnelly declares that he has no conflict of interest.

Ethical approval: Ethical approval was not required because of the review nature of the research. This article does not contain any studies with animals performed by any of the authors.

\section{References}

1. Ferlay J, Soerjomataram I, Ervik M, Dikshit R, Eser S, Mathers C, et al. GLOBOCAN 2012 v1.0, Cancer Incidence and Mortality Worldwide: IARC CancerBase. No. 11. Lyon, Fr. Int. Agency Res. Cancer. 2013.

2. Beatty L, Oxlad M, Koczwara B, Wade TD. The psychosocial concerns and needs of women recently diagnosed with breast cancer: a qualitative study of patient, nurse and volunteer perspectives. Heal. Expect. 2008;11:331-42.

3. Lindberg P, Koller M, Steinger B, Lorenz W, Wyatt JC, Inwald EC, et al. Breast cancer survivors' recollection of their illness and therapy seven years after enrolment into a randomised controlled clinical trial. BMC Cancer. 2015;15:554.

4. Treanor, Donnelly M. The late effects of cancer and cancer treatment: a rapid review. J. Community Support. Oncol. 2014;12:137-48.

5. Treanor C, Santin O, Mills M, Donnelly M. Cancer survivors with self-reported late effects: Their health status, care needs and service utilisation. Psychooncology. 2013;22:2428-35.

6. Treanor C, Donnelly M. Late effects of cancer and cancer treatment - the perspective of the patient. Support. Care Cancer. 2016;24:337-46. 
7. Silver JK. Cancer Prehabilitation and its Role in Improving Health Outcomes and Reducing Health Care Costs. Semin. Oncol. Nurs. 2015;31:13-30.

8. Silver JK, Baima J. Cancer prehabilitation: an opportunity to decrease treatment-related morbidity, increase cancer treatment options, and improve physical and psychological health outcomes. Am. J. Phys. Med. Rehabil. $2013 ; 92: 715-27$.

9. Higgins JPT, Green S. Cochrane Handbook for Systematic Reviews of Interventions.Version 5. The Cochrane Collaboration; 2011.

10. Moher D, Liberati A, Tetzlaff J, Altman DG. Preferred Reporting Items for Systematic Reviews and MetaAnalyses: The PRISMA Statement. PLoS Med. 2009;6:e1000097.

11. Silver JK, Baima J. Cancer Prehabilitation. Am. J. Phys. Med. Rehabil. 2013;92:715-27.

12. Benzo R, Wigle D, Novotny P, Wetzstein M, Nichols F, Shen RK, et al. Preoperative pulmonary rehabilitation before lung cancer resection: Results from two randomized studies. Lung Cancer. 2011;74:441-5. 13. Parker PA, Pettaway CA, Babaian RJ, Pisters LL, Miles B, Fortier A, et al. The effects of a presurgical stress management intervention for men with prostate cancer undergoing radical prostatectomy. J. Clin. Oncol. 2009;27:3169-76.

14. Cohen L, Parker PA, Vence L, Savary C, Kentor D, Pettaway C, et al. Presurgical Stress Management Improves Postoperative Immune Function in Men With Prostate Cancer Undergoing Radical Prostatectomy. Psychosom. Med. 2011;73:218-25.

15. Purcell A, Fleming J, Burmeister B, Bennett S, Haines T. Is education an effective management strategy for reducing cancer-related fatigue? Support. Care Cancer. 2011;19:1429-39.

16. Parekh AR, Feng MI, Kirages D, Bremner H, Kaswick J, Aboseif S. The role of pelvic floor exercises on post-prostatectomy incontinence. J. Urol. 2003;170:130-3.

17. Lai Y, Huang J, Yang M, Su J, Liu J, Che G. Seven-day intensive preoperative rehabilitation for elderly patients with lung cancer: a randomized controlled trial. J. Surg. Res. 2017;209:30-6.

18. Licker M, Karenovics W, Diaper J, Frésard I, Triponez F, Ellenberger C, et al. Short-Term Preoperative High-Intensity Interval Training in Patients Awaiting Lung Cancer Surgery: A Randomized Controlled Trial. J. Thorac. Oncol. 2017;12:323-33. 
19. Tienforti D, Sacco E, Marangi F, D’Addessi A, Racioppi M, Gulino G, et al. Efficacy of an assisted lowintensity programme of perioperative pelvic floor muscle training in improving the recovery of continence after radical prostatectomy: A randomized controlled trial. BJU Int. 2012;110:1004-10.

20. Cho HSM, Davis GC, Paek JE, Rao R, Zhao H, Xie XJ, et al. A randomised trial of nursing interventions supporting recovery of the postmastectomy patient. J. Clin. Nurs. 2013;22:919-29.

21. Burgio KL, Goode PS, Urban DA, Umlauf MG, Locher JL, Bueschen A, et al. Preoperative biofeedback assisted behavioral training to decrease post-prostatectomy incontinence: a randomized, controlled trial. J. Urol. 2006;175:196-201.

22. Pehlivan E, Turna A, Gurses A, Gurses HN. The effects of preoperative short-term intense physical therapy in lung cancer patients: A randomized controlled trial. Ann. Thorac. Cardiovasc. Surg. 2011;17:461-8.

23. Burton M, Parker R, Farrell A. A randomized controlled trial of presurgical psychological preparation for mastectomy. Psychooncology 1995;4:1-19.

24. Jensen B, Petersen A, Jensen J, Laustsen S, Borre M. Efficacy of a multiprofessional rehabilitation programme in radical cystectomy pathways: A prospective randomized controlled trial. Scand. J. Urol. $2015 ; 49: 133-41$.

25. Stefanelli F, Meoli I, Cobuccio R, Curcio C, Amore D, Casazza D, et al. High-intensity training and cardiopulmonary exercise testing in patients with chronic obstructive pulmonary disease and non-small-cell lung cancer undergoing lobectomy. Eur. J. Cardio-Thoracic Surg. 2013;44:e260-5.

26. Wall LM. Changes in hope and power in lung cancer patients who exercise. Nurs. Sci. Q. 2000;13:234-42.

27. Larson MR, Duberstein PR, Talbot NL, Caldwell C, Moynihan J a. A presurgical psychosocial intervention for breast cancer patients. psychological distress and the immune response. J. Psychosom. Res. 2000;48:187-94.

28. Centemero A, Rigatti L, Giraudo D, Lazzeri M, Lughezzani G, Zugna D, et al. Preoperative pelvic floor muscle exercise for early continence after radical prostatectomy: a randomised controlled study. Eur. Urol. 2010;57:1039-43.

29. Garssen B, Boomsma M, De Jager Meezenbroek E, Porsild T, Berkhof J, Berbee M, et al. Stress management training for breast cancer surgery patients. Psychooncology. 2013;22:572-80.

30. Gillis C, Li C, Lee L, Awasthi R, Augustin B, Gamsa A, et al. Prehabilitation versus Rehabilitation. 
Anesthesiology. 2015;122:1438.

31. Harada H, Yamashita Y, Misumi K, Tsubokawa N, Nakao J, Matsutani J, et al. Multidisciplinary TeamBased Approach for Comprehensive Preoperative Pulmonary Rehabilitation Including Intensive Nutritional Support for Lung Cancer Patients. PLoS One. 2013;8:e59566.

32. Pouwels S, Fiddelaers J, Teijink JAW, Woorst JF Ter, Siebenga J, Smeenk FWJM. Preoperative exercise therapy in lung surgery patients: A systematic review. Respir. Med. 2015;109:1495-504.

33. Cavalot AL, Ricci E, Schindler A, Roggero N, Albera R, Utari C, et al. The importance of preoperative swallowing therapy in subtotal laryngectomies. Otolaryngol. - Head Neck Surg. 2009;140:822-5.

34. Carroll WR, Locher JL, Canon CL, Bohannon IA, McColloch NL, Magnuson JS. Pretreatment Swallowing Exercises Improve Swallow Function After Chemoradiation. Laryngoscope 2008;118:39-43.

35. Inoue J, Ono R, Makiura D, Kashiwa-Motoyama M, Miura Y, Usami M, et al. Prevention of postoperative pulmonary complications through intensive preoperative respiratory rehabilitation in patients with esophageal cancer. Dis. Esophagus. 2013;26:68-74.

36. Bade BC, Thomas DD, Scott JB, Silvestri GA. Increasing Physical Activity and Exercise in Lung Cancer: Reviewing Safety, Benefits, and Application. J. Thorac. Oncol. 2015;10:861-71.

37. Jensen BT, Laustsen S, Jensen JB, Borre M, Petersen AK. Exercise-based pre-habilitation is feasible and effective in radical cystectomy pathways—-secondary results from a randomized controlled trial. Support. Care Cancer. 2016;24:1-7.

38. Dronkers JJ, Lamberts H, Reutelingsperger IMMD, Naber RH, Dronkers-Landman CM, Veldman a, et al. Preoperative therapeutic programme for elderly patients scheduled for elective abdominal oncological surgery: a randomized controlled pilot study. Clin. Rehabil. 2010;24:614-22.

39. Dunne DFJ, Jack S, Jones RP, Jones L, Lythgoe DT, Malik HZ, et al. Randomized clinical trial of prehabilitation before planned liver resection. Br. J. Surg. 2016;504-12.

40. Carli F, Brown R, Kennepohl S. Prehabilitation to enhance postoperative recovery for an octogenarian following robotic-assisted hysterectomy with endometrial cancer. Can. J. Anesth. 2012;59:779-84.

41. Department of Health, Macmillan Cancer Support, NHS Improvement. Living with and beyond cancer: Taking action to improve outcomes. London; 2013. 
42. Focht KL, Simpson K, Martin-Harris B. Business Case for Pretreatment Swallowing Exercises. Otolaryngol.

-- Head Neck Surg. 2011;145:P161-2. 